\title{
PTSD—more complicated on second look
}

\section{Richard Fielding and Wendy W. T. Lam}

We read with interest the News \& Views article by Palesh and Koopman (Posttraumatic stress disorder-prevalent and persistent. Nat. Rev. Clin. Oncol. 10, 252$254 ; 2013)^{1}$ that focused on a recent report by vin-Raviv et al. ${ }^{2}$ on racial differences in post-traumatic stress disorder (PTSD). Both the commentary and the underlying paper unquestioningly present the common-sense picture that, for most women with breast cancer, distress is highest at or around the time of diagnosis, but steadily declines thereafter. ${ }^{3,4}$ This view has prevailed in the literature for over 15 years. ${ }^{5}$

However, all but a handful of the studies describing the psychosocial outcomes in patients with cancer used whole-group analyses of longitudinal data, which-in reality-were serial cross-sectional studies. Although simply, and possibly correctly, reporting declining proportions of distressed patients at different time points, these proportionate studies misrepresent what is a more complex response. Few studies have adopted different analytical approaches that decompose samples into smaller subsets of trajectories. ${ }^{6-8}$ These reports suggest a very different picture to that implied by the prevailing view. Firstly, most patients with cancer experience persistent low levels of distress (or PTSD) across the 6 months following breast (or other) cancer (or other traumatic) ${ }^{9}$ diagnoses. There are critical time points-such as diagnosis, surgery and treatment cessation-but acute distress responses probably quickly revert. Second, only a small minority of patients (typically around 20\%) follow the 'classic' high-to-low pattern of distress that begins with a high level of stress and declines steadily over time. In fact, a comparable proportion (20\%) of patients begin with low distress that increases over time, typically at 1-3 months, but then declines by 8 months. Furthermore, approximately $12-25 \%$ of patients experience persistently high levels of distress that continue for at least 8 months and for whom, 6 years later, psychosocial outcomes remain poor compared with their peers. ${ }^{10}$ This pattern is consistent in other potentially traumatic health conditions, such as spinal cord injury and severe acute respiratory syndrome. ${ }^{9}$

A second problem overlooked by vin Raviv et al. ${ }^{2}$ is the recognition that 'Asian' is a far too gross a generalization to be informative. Were these women mostly of Indian, Chinese, Japanese, Filipino, Malay, Thai, Viet, Korean, Indonesian or other ethnicities? Between Hong Kong and Taiwanese Chinese and Japanese patients with colorectal cancer, we have observed markedly different levels of psychological unmet need, ${ }^{11}$ corresponding to the distress typified by what vin-Raviv et al. ${ }^{2}$ (and Palesh and Koopman) ${ }^{1}$ quantify as PTSD.

Additionally, vin-Raviv et al. ${ }^{2}$ used the Impact of Events Scale (IES), which does not provide a definitive diagnosis of PTSD. Although not discussed by Palesh and Koopman, ${ }^{1}$ the IES has been criticized $^{12}$ for being nonspecific, omitting symptom clusters important in PTSD diagnosis and specific symptoms that include hyperarousal. To use a measure of what amounts to general distress to define PTSD risks extensively over-pathologizing what might be considered a 'normal' distress response. ${ }^{12}$

Finally, Palesh and Koopman ${ }^{1}$ recommend screening for PTSD, but ignore the thorny questions of when and for whom. Different people react at different time points and, unless screening is done constantly, it will be largely ineffective. Given the high cost of psychological interventions, most patients receiving a positive screen might be further burdened by more expensive, and possibly unnecessary, treatments. Unless we can distinguish those likely to experience chronic high distress from those on other distress trajectories, PTSD screening will inevitably be an extremely costly endeavor, which would be a more important trauma for many.

Oncologists must understand these important factors when presented with a distressed patient newly diagnosed with cancer. Patients might be distressed. However, not all distress signifies PTSD and not all distress requires an intervention. Care decisions will likely be affected by a distressed patient and, therefore, physicians must be informed.
Centre for Psycho-Oncology Research and Training, School of Public Health, 5/F W. M. W. Mong Building, The University of Hong Kong, 21 Sassoon Road, Pokfulam, Hong Kong, China (R. Fielding, W. W. T. Lam). Correspondence to: W. W. T. Lam wwtlam@hku.hk

\section{Competing interests}

R. Fielding has received speaker's fees from the following companies: Macquaries Securities and MundiPharma. W. W. T. Lam has received speaker's fees from the following companies: MundiPharma and Novartis.

1. Palesh, O. \& Koopman, C. Breast cancer: posttraumatic stress disorder-prevalent and persistent. Nat. Rev. Clin. Oncol. 10, 252-254 (2013).

2. vin-Raviv, N. et al. Racial disparities in posttraumatic stress after diagnosis of localized breast cancer: the BQUAL study. J. Natl Cancer Inst. 105, 563-572 (2013).

3. Burgess, C. et al. Depression and anxiety in women with early breast cancer: five year observational cohort study. BMJ 330, 702 (2005).

4. Epping-Jordan, J. E. et al. Psychological adjustment in breast cancer: processes of emotional distress. Health Psychol. 18, 315-326 (1999).

5. Tjemsland, L., Søreide, J. A. \& Malt, U. F. Traumatic distress symptoms in early breast cancer. II: outcome six weeks post surgery. Psychooncology 5, 295-303 (1996).

6. Helgeson, V. S., Snyder, P. \& Seltman, H. Psychological and physical adjustment to breast cancer over 4 years: identifying distinct trajectories of change. Health Psychol. 23, 3-15 (2004).

7. Deshields, T., Tibbs, T., Fan, M. Y. \& Taylor, M. Differences in patterns of depression after treatment for breast cancer. Psychooncology 15, 398-406 (2006).

8. Lam, W. W. et al. Trajectories of psychological distress among Chinese women diagnosed with breast cancer. Psychooncology 19 1044-1051 (2010).

9. Bonanno, G. A. et al. Psychological resilience and dysfunction among hospitalized survivors of the SARS epidemic in Hong Kong: a latent class approach. Health Psychol. 27, 659-667 (2008).

10. Lam, W. W., Shing, Y. T., Bonanno, G. A., Mancini, A. D. \& Fielding, R. Distress trajectories during the first year following diagnosis of breast cancer in relation to 6-years survivorship. Psychooncology 21, 90-99 (2011).

11. Fielding, R. et al. Attributing variance in supportive care needs during cancer: cultureservice and individual differences before clinical factors. PLOS ONE 8, e65099 (2013).

12. Coyne, J. Cancer as a psychological trauma. Mindthebrain PLoS Blogs [online], http:// blogs.plos.org/mindthebrain/2013/04/15/ cancer-as-a-psychological-trauma (2013). 